\title{
Characteristic properties of FPTP systems
}

\author{
Eliora van der Hout • Harrie de Swart
}

Published online: 22 May 2009

(C) The Author(s) 2009. This article is published with open access at Springerlink.com

\begin{abstract}
In this article, we model FPTP systems as social preference rules and give two characterizations. We show that a social preference rule is an FPTP system if, and only if, it satisfies the axioms of subset consistency, district consistency, subset cancellation, and district cancellation. The second characterization consists of the axioms of subset consistency, subset anonymity, neutrality, topsonlyness, Pareto optimality, district consistency and district cancellation. The characterizations give us an opportunity to compare the characteristic properties of FPTP systems to the characteristic properties that we found for list systems of proportional representation (list PR systems) in Hout et al. (Social Choice and Welfare, 27:459-475, 2006), where we modelled those systems also as social preference rules. We find that consistency and anonymity distinguish list PR systems from FPTP systems. On the other hand, it is district cancellation that distinguishes FPTP systems from list PR systems.
\end{abstract}

Keywords Social choice $\cdot$ FPTP systems $\cdot$ Plurality ranking rule

\section{Introduction}

In Hout et al. (2006), we gave characterizations of the list systems of proportional representation (list PR systems) that are used in most West European countries to choose representatives. We modelled these systems as social preference rules. These are rules that assign to each combination of individual preference orderings of the parties a social ordering of these parties, as it may be reflected in the number of seats that each party is assigned. Since in a list PR system, parties are assigned a number of seats in parliament that is proportional to the number of votes they received, we

E. van der Hout $\cdot$ H. de Swart $(\varangle)$

Department of Philosophy, Tilburg University, P.O. Box 90153, 5000 LE Tilburg, The Netherlands

e-mail: H.C.M.deSwart@uvt.nl 
modelled it as a social preference rule that ranks a party higher (assigns more seats) when it is the first preference of more voters (receives more first votes). This rule is known as the plurality ranking rule.

A second class of rules that are used in Western democracies to choose representatives, are the so-called first past the post (FPTP) systems (Lijphart 1994; Farrell 1997). In these systems, the electorate is assigned to a large number of districts in each of which one single representative is chosen using the plurality rule. Therefore, in each district the candidate who received the most votes wins the single seat. This means that a party $x$ receives more seats than a party $y$ if the number of districts in which a candidate of party $x$ wins a plurality of the votes is larger than the number of districts in which a candidate of party $y$ wins a plurality of the votes. Because of that, in this article, we model FPTP systems as social preference rules that rank a party $x$ before a party $y$ (respectively equal to $y$ ) if the number of constituencies, in which $x$ is ranked first by the largest number of voters, is larger than (respectively equal to) the number of constituencies in which $y$ is ranked first by the largest number of voters.

A good example of an FPTP system is the British electoral system. The British electorate is assigned to approximately 659 districts, in each of which one single representative is chosen using the plurality rule. Other examples of countries that use such a single-member plurality system are the U.S. and Canada.

We give two characterizations of the FPTP systems, using axioms that resemble the axioms that were used in Hout (2006). These characterizations give us an opportunity, not only to evaluate FPTP systems on the basis of their characteristic properties, but also to compare them to the characteristic properties for list PR systems that we found in Hout (2006). In the final sections of this article, we make such a comparison.

List PR systems and FPTP systems embody two different views on representation, which are distinguished by Pitkin (1967). According to descriptive representation that is associated with systems of proportional representation, parliament should be representative in the sense that it includes the same proportion of each relevant subgroup as the population at large. Systems like the British system, on the other hand, are associated with substantive representation. In this conception, representatives are agents that act on behalf of others. Parliament need not contain the same subgroups as the population, but needs to act on behalf of the electorate. Thus, the two categories of electoral systems seem to have been designed with different goals. Because of this, both kinds of systems, i.e. both sets of characteristic axioms, may be defendable, although based on two different views on representation.

\section{Preliminaries}

Let $V$ be a finite set of voters. Since in practice not all voters will cast their vote, we want our social preference rule to be applicable to finite, nonempty subsets $I$ of $V$. The set of alternatives, in this case (candidates of) political parties, will be identified with $A . R \subseteq A \times A$ will be conceived of as a preference relation. This means that the statement $(x, y) \in R$ will be interpreted in the sense that $x \in A$ is at least as good as $y \in A$. A preference relation $R$ is a weak ordering on $A$ if $R$ is complete and transitive. 
$R$ is a linear ordering on $A$ if $R$ is complete, transitive and antisymmetric. The set of all weak orderings on $A$ will be denoted by $W(A)$ and the set of all linear orderings on $A$ will be denoted by $L(A)$.

We assume that each individual $i \in V$ orders the parties in a strict way, i.e., for all $i \in V, R_{i} \in L(A)$. For each $I \subseteq V$, a specific combination of linear orderings on $A$ of the individuals in $I$, the so-called preference profile on $I$, can thus be formulated as a function $c: I \rightarrow L(A)$. The set of all preference profiles on $I$ is equal to $L(A)^{I}$. For the linear ordering of individual $i \in I$ in preference profile $c$ on $I$, we will write $c_{i} . x \succ_{c_{i}} y$ denotes that $x$ occurs before $y$ in the individual ordering $c_{i}$ of voter $i \in I$. $t\left(c_{i}\right)=x$ denotes that $x$ is the top of $c_{i}$. For $c \in L(A)^{I}, \pi_{c}(x)=\left|\left\{i \in I \mid t\left(c_{i}\right)=x\right\}\right|$, i.e., the number of voters in $I$ who have $x$ as their first preference.

The set of individuals may be divided into constituencies. This fact can be formalized as follows. Let $\Delta=\left\{\delta_{1}, \ldots, \delta_{m}\right\}$ be a partition of the set of voters $V$, i.e., (i) for all $\delta \in \Delta, \delta \subseteq V$; (ii) for all $\delta^{\prime}, \delta^{\prime \prime} \in \Delta$, if $\delta^{\prime} \neq \delta^{\prime \prime}$, then $\delta^{\prime} \cap \delta^{\prime \prime}=\varnothing$; (iii) $\cup\{\delta \mid \delta \in \Delta\}=V$. The partition $\Delta$ of $V$ will be interpreted as a division of the voters in $V$ into constituencies. For each $I \subseteq V, \Delta$ induces a partition $\Delta^{I}$ of $I$; $\Delta^{I}=\left\{\delta_{1} \cap I, \ldots, \delta_{m} \cap I\right\}$.

For a profile $c \in L(A)^{I}$, a partition $\Delta$ of $V$ induces the so-called district profiles on each $\delta \cap I$, that can be formulated as functions $c_{\delta}: \delta \cap I \rightarrow L(A)$, where $c_{\delta}$ is the restriction of $c$ to $\delta \cap I$. The number of individuals in $\delta \cap I$ with $x$ at the top of their individual preference ranking will be denoted by $\pi_{c_{\delta}}(x)$. Therefore, for $\delta \in \Delta$, $I \subseteq V$, and $x \in A$,

$$
\pi_{c_{\delta}}(x)=\left|\left\{i \in \delta \cap I \mid t\left(c_{i}\right)=x\right\}\right| .
$$

The number of constituencies in which $x$ is ranked first by the largest number of voters is denoted by $\tau_{c}^{\Delta}(x)$. Formally, for $I \subseteq V, \delta \in \Delta$, and $x \in A$, we define ' $x$ is preferred most in district $\delta$ given profile $c \in L(A)^{I}$, by $\pi_{c_{\delta}}(x) \geq \pi_{c_{\delta}}(z)$ for all $z \in A$. For $I \subseteq V, c \in L(A)^{I}$, and $x \in A$,

$$
\tau_{c}^{\Delta}(x)=\mid\left\{\delta \cap I \in \Delta^{I} \mid x \text { is preferred most in } \delta \cap I \text { given } c\right\} \mid .
$$

Given a set of voters $I$ and a partition $\Delta$ of $V$, a social preference rule is a function

$$
F_{\Delta}: L(A)^{I} \rightarrow W(A) .
$$

Thus, for each finite set $I$ of voters, and for every partition $\Delta$ of $V$, a social preference rule assigns to each preference profile $c \in L(A)^{I}$ a weak (social) ordering $F_{\Delta}(c)$ of the parties. By definition,

$$
t\left(F_{\Delta}(c)\right)=\left\{x \in A \mid x \text { is a top element of } F_{\Delta}(c)\right\} .
$$

Typically, $t\left(F_{\Delta}(c)\right)$ will contain just one element, but society might be indifferent with respect to two or more parties.

Since in FPTP systems every voter is allowed to cast one single vote, a voter can only vote for the party that is ordered first in his individual preference ordering. A 
voter may, of course, strategically misrepresent his preferences and vote, for example, for the party that is ordered second in his individual preference ordering. However, in this article, we only describe how the individual linear orderings, manipulated or not, are transformed to a (weak) social ordering over the parties. After the votes have been cast, they are summarized, and party $x$ receives more seats than party $y$ if the number of districts in which a candidate of party $x$ wins a plurality of the votes is larger than the number of districts in which a candidate of party $y$ wins a plurality of the votes. Hence, we may model FPTP systems as social preference rules that rank a party $x$ before a party $y$ (respectively equal to $y$ ) if the number of constituencies in which $x$ is ranked first by the largest number of voters is larger than (respectively equal to) the number of constituencies in which $y$ is ranked first by the largest number of voters.

Definition $1\left(\mathrm{FPTP}_{\Delta}\right)$ The function $\mathrm{FPTP}_{\Delta}: L(A)^{I} \rightarrow W(A)$, for $I \subseteq V$, is defined by

$$
x \succeq_{F P T P_{\Delta(c)}} y \text { iff } \tau_{c}^{\Delta}(x) \geq \tau_{c}^{\Delta}(y) .
$$

Although we define both list PR systems and FPTP systems as social preference rules, the definition of a social preference rule we use in both characterizations is slightly different. A list PR system is defined as a function $F: L(A)^{I} \rightarrow W(A)$, for each $I \subseteq V$, while an FPTP system was defined as a function $F_{\Delta}: L(A)^{I} \rightarrow W(A)$ for each $I \subseteq V$, where $\Delta$ is a partition of $V$. However, list PR systems can also be applied to sets of voters that are assigned to constituencies. List PR systems differ from FPTP systems in the fact that the former do not make use of this assignment to constituencies when assigning a social ordering to a preference profile. Therefore, for list PR systems, we can also write $F_{\Delta}$ instead of $F$.

Definition 2 (Plurality ranking rule) The plurality ranking rule $P l: L(A)^{I} \rightarrow W(A)$, for $I \subseteq V$, is defined by:

$$
x \succeq_{P l(c)} y \text { iff } \pi_{c}(x) \geq \pi_{c}(y) .
$$

\section{Axioms for social preference rules}

By consistency (Young 1974; Nitzan and Rubinstein 1981) of a social preference rule $F_{\Delta}$, we mean that if two disjoint sets of voters $I$ and $J$ both socially prefer $x$ to $y$ using $F_{\Delta}$, their union should also socially prefer $x$ to $y$ using $F_{\Delta}$.

Definition 3 (Consistency) A social preference rule $F_{\Delta}$ is consistent if whenever $c \in L(A)^{I}, c^{\prime} \in L(A)^{J}$ are preference profiles for disjoint sets of voters $I \subseteq V$ and $J \subseteq V$ and $c+c^{\prime}$ is the profile on $I \cup J$ that corresponds with $c$ on $I$ and with $c^{\prime}$ on $J$, for all $x, y \in A$ :

$$
\text { if } x \succ_{F_{\Delta}(c)} y \text { and } x \succeq_{F_{\Delta}\left(c^{\prime}\right)} y \text {, then } x \succ_{F_{\Delta}\left(c+c^{\prime}\right)} y \text {. }
$$

Clearly, F PT $P_{\Delta}$ is not consistent, as the following example shows. 
Counterexample $1 \delta_{1}=\{1,2\}, \delta_{2}=\{3,4,5\}, I=\{1,2,3\}, J=\{4,5\}$.

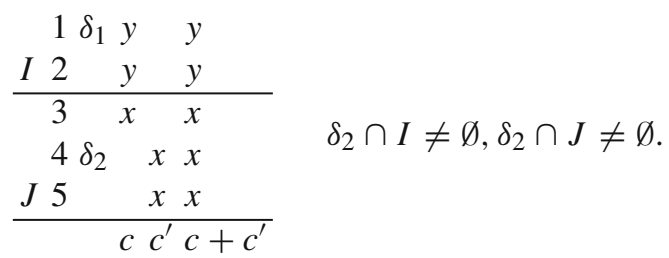

$x \sim_{F P T P_{\Delta}(c)} y$ and $x \succ_{F P T P_{\Delta}\left(c^{\prime}\right)} y$, but not $x \succ_{F P T P_{\Delta}\left(c+c^{\prime}\right)} y$. This also holds if $\delta_{1}=\{1,2,4\}$ and $\delta_{2}=\{3,5\}$, in which case the intersection of both $I$ and $J$ with each of $\delta_{1}, \delta_{2}$ is non-empty.

However, if one demands that every $\delta \in \Delta$ having a non-empty intersection with $I$ does have an empty intersection with $J$, then consistency is preserved, as will be shown in Proposition 2.

FPTP $_{\triangle}$ also does satisfy subset consistency. Subset consistency relates the choices made by disjoint subsets of a particular constituency, to choices made by their union. It requires that if two sets of voters within the same constituency use the same social preference rule and the tops of the social orderings they choose have at least one element in common, then the top of the social ordering that is chosen by their union should contain exactly these shared elements.

Definition 4 (Subset Consistency) Let $I^{\delta} \subseteq \delta \subseteq V$ and $J^{\delta} \subseteq \delta \subseteq V$ be disjoint subsets of one single constituency $\delta$. A social preference rule is subset consistent if, whenever $c_{\delta} \in L(A)^{I^{\delta}}$ and $c_{\delta}^{\prime} \in L(A)^{J^{\delta}}$ are preference profiles for $I^{\delta}$ and $J^{\delta}$, and $c_{\delta}+c_{\delta}^{\prime}$ is the profile on $I^{\delta} \cup J^{\delta}$ that corresponds with $c_{\delta}$ on $I^{\delta}$ and with $c_{\delta}^{\prime}$ on $J^{\delta}, t\left(F_{\Delta}\left(c_{\delta}\right)\right) \cap$ $t\left(F_{\Delta}\left(c_{\delta}^{\prime}\right)\right) \neq \emptyset$ implies $t\left(F_{\Delta}\left(c_{\delta}+c_{\delta}^{\prime}\right)\right)=t\left(F_{\Delta}\left(c_{\delta}\right)\right) \cap t\left(F_{\Delta}\left(c_{\delta}^{\prime}\right)\right)$.

Proposition $1 \mathrm{FPTP}_{\Delta}$ satisfies subset consistency.

Proof Let $\delta \in \Delta$, let $c_{\delta} \in L(A)^{I^{\delta}}$ and $c_{\delta}^{\prime} \in L(A)^{J^{\delta}}$ be preference profiles for disjoint sets of voters $I^{\delta} \subseteq \delta$ and $J^{\delta} \subseteq \delta$, and $c_{\delta}+c_{\delta}^{\prime}$ be the profile on $I^{\delta} \cup J^{\delta}$ that corresponds with $c_{\delta}$ on $I^{\delta}$ and with $c_{\delta}^{\prime}$ on $J^{\delta}$. Let $t\left(F P T P_{\Delta}\left(c_{\delta}\right)\right) \cap t\left(F P T P_{\Delta}\left(c_{\delta}^{\prime}\right)\right) \neq \emptyset$. Then, for all $x \in t\left(F P T P_{\Delta}\left(c_{\delta}\right)\right) \cap t\left(F P T P_{\Delta}\left(c_{\delta}^{\prime}\right)\right), \tau_{c_{\delta}}^{\Delta}(x)=1$ and $\tau_{c_{\delta}^{\prime}}^{\Delta}(x)=1$ and thus $\pi_{c_{\delta}}(x) \geq \pi_{c_{\delta}}(y)$ for all $y \in A$ and $\pi_{c_{\delta}^{\prime}}(x) \geq \pi_{c_{\delta}^{\prime}}(y)$ for all $y \in A$. Since $I^{\delta} \cap J^{\delta}=\emptyset$, for all $x \in A, \pi_{c_{\delta}+c_{\delta}^{\prime}}(x)=\pi_{c_{\delta}}(x)+\pi_{c_{\delta}^{\prime}}(x)$. Thus, if $\tau_{c_{\delta}}^{\Delta}(x)=1$ and $\tau_{c_{\delta}^{\prime}}^{\Delta}(x)=1$, then $\tau_{c_{\delta}+c_{\delta}^{\prime}}^{\Delta}(x)=1$. So, if $x \in t\left(F P T P_{\Delta}\left(c_{\delta}\right)\right) \cap t\left(F P T P_{\Delta}\left(c_{\delta}^{\prime}\right)\right)$, then $x \in t\left(F P T P_{\Delta}\left(c_{\delta}+c_{\delta}^{\prime}\right)\right)$.

Conversely, suppose $z \in t\left(F P T P\left(c_{\delta}+c_{\delta}^{\prime}\right)\right)$, thus $\tau_{c_{\delta}+c_{\delta}^{\prime}}^{\Delta}(z)=1$. Let $x \in t\left(F P T P_{\Delta}\right.$ $\left.\left(c_{\delta}\right)\right) \cap t\left(F P T P_{\Delta}\left(c_{\delta}^{\prime}\right)\right) \neq \emptyset$, thus $\tau_{c_{\delta}}^{\Delta}(x)=1$ and $\tau_{c_{\delta}^{\prime}}^{\Delta}(x)=1$. If $z \notin t\left(F P T P_{\Delta}\left(c_{\delta}\right)\right)$ or $z \notin t\left(F P T P_{\Delta}\left(c_{\delta}^{\prime}\right)\right)$, then $\tau_{c_{\delta}}^{\Delta}(z)=0$ or $\tau_{c_{\delta}^{\prime}}^{\Delta}(z)=0$. So, $\pi_{c_{\delta}}(x)>\pi_{c_{\delta}}(z)$ or $\pi_{c_{\delta}^{\prime}}(x)>$ $\pi_{c_{\delta}^{\prime}}(z)$. Consequently, $\pi_{c_{\delta}+c_{\delta}^{\prime}}(x)>\pi_{c_{\delta}+c_{\delta}^{\prime}}(z)$ and, thus, $\tau_{c_{\delta}+c_{\delta}^{\prime}}^{\Delta}(z)=0$. This contradicts $z \in t\left(F P T P_{\Delta}\left(c_{\delta}+c_{\delta}^{\prime}\right)\right)$. Hence, $z \in t\left(F P T P_{\Delta}\left(c_{\delta}\right)\right)$ and $z \in t\left(F P T P_{\Delta}\left(c_{\delta}^{\prime}\right)\right)$. 
The second new axiom we introduce here is district consistency. District consistency is similar to the usual consistency, with the proviso that the two sets of voters in question are not only disjoint, but both do not contain elements of a same constituency either. Note that in our counterexample 1 above, showing that $F P T P_{\Delta}$ is not consistent, this proviso is violated: in this example, both $I$ and $J$ contain elements of the constituency $\delta_{2}$.

Definition 5 (District Consistency). A social preference rule $F_{\Delta}$ is district consistent if, whenever $c \in L(A)^{I}$, and $c^{\prime} \in L(A)^{J}$ are preference profiles for disjoint sets of voters $I \subseteq V$ and $J \subseteq V$, such that for all $\delta \in \Delta, \delta \cap I \neq \emptyset$ implies $\delta \cap J=\emptyset$, and $c+c^{\prime}$ is the profile on $I \cup J$, that corresponds with $c$ on $I$ and with $c^{\prime}$ on $J$, for all $x, y \in A$ : if $x \succ_{F_{\Delta}(c)} y$ and $x \succeq_{F_{\Delta}\left(c^{\prime}\right)} y$, then $x \succ_{F_{\Delta}\left(c+c^{\prime}\right)} y$.

Proposition $2 \mathrm{FPTP}_{\Delta}$ satisfies district consistency.

Proof Let $I \subseteq V$ and $J \subseteq V$ be two sets of voters such that for all $\delta \in \Delta, \delta \cap I \neq \emptyset$ implies $\delta \cap J=\emptyset$. Let $c \in L(A)^{I}$, and $c^{\prime} \in L(A)^{J}$ be preference profiles for $I \subseteq V$ and $J \subseteq V$ and let $c+c^{\prime}$ be the profile on $I \cup J$, that corresponds with $c$ on $I$ and with $c^{\prime}$ on $J$. Let $x, y \in A$ and let $x \succ_{F P T P}(c) y$ and $x \succeq_{F P T P_{\Delta}\left(c^{\prime}\right)} y$. Then $\tau_{c}^{\Delta}(x)>\tau_{c}^{\Delta}(y)$ and $\tau_{c^{\prime}}^{\Delta}(x) \geq \tau_{c^{\prime}}^{\Delta}(y)$. Since $I \cap J=\emptyset$ and since for all $\delta \in \Delta, \delta \cap I \neq \emptyset$ implies $\delta \cap J=\emptyset$, for all $x \in A, \tau_{c+c^{\prime}}^{\Delta}(x)=\tau_{c}^{\Delta}(x)+\tau_{c^{\prime}}^{\Delta}(x)$. Hence, $\tau_{c+c^{\prime}}^{\Delta}(x)>\tau_{c+c^{\prime}}^{\Delta}(y)$. Hence, $x \succ_{F P T P_{\Delta}\left(c+c^{\prime}\right)} y$.

The third axiom in the characterization of $F P T P_{\Delta}$ resembles the FS-cancellation axiom in Hout (2006). Here, FS refers to First Score in the sense that only the first or top preferences of the voters are taken into account. In this sense, this notion is different from the original notion in Young (1974); Nitzan and Rubinstein (1981).

Definition 6 (FS Cancellation). A social preference rule $F_{\Delta}$ has the FS cancellation property if, for all $I \subseteq V$, for every $c \in L(A)^{I}$, and for all $x, y \in A$, if $\pi_{c}(x)=\pi_{c}(y)$, then $x \sim_{F_{\Delta}(c)} y$.

$\mathrm{FPTP}_{\Delta}$ does not satisfy FS cancellation, as the following example shows.

Counterexample 2 Let $A=\{x, y, z\}, I=V=\{1, \ldots, 6\}, \Delta=\left\{\delta_{1}, \delta_{2}\right\}$ with $\delta_{1}=$ $\{1,2,3\}$ and $\delta_{2}=\{4,5,6\}$. Suppose $t\left(c_{1}\right)=t\left(c_{2}\right)=x, t\left(c_{3}\right)=t\left(c_{4}\right)=y$ and $t\left(c_{5}\right)=t\left(c_{6}\right)=z$. Then $\pi_{c}(x)=\pi_{c}(y)=2$, but not $x \sim_{F P T P_{\Delta}}(c) y$, since $x$ wins in district $\delta_{1}$, while $y$ wins in no district.

Subset cancellation demands that whenever a set of voters is a subset of one single constituency and all alternatives that receive a nonzero vote total tie, the social preference rule should rank this whole set of alternatives first.

Definition 7 (Subset Cancellation) A social preference rule $F_{\Delta}$ has the subset cancellation property if, for all $\delta \in \Delta$, for all $I^{\delta} \subseteq \delta \subseteq V$, and for every $c_{\delta} \in L(A)^{I^{\delta}}$, if for all parties $x, y \in A$ with $\pi_{c_{\delta}}(x) \neq 0$ and $\pi_{c_{\delta}}(y) \neq 0, \pi_{c_{\delta}}(x)=\pi_{c_{\delta}}(y)$, then $t\left(F_{\Delta}\left(c_{\delta}\right)\right)=\left\{x \in A \mid \pi_{c_{\delta}}(x) \neq 0\right\}$.

Proposition $3 \mathrm{FPTP}_{\Delta}$ satisfies subset cancellation. 
Proof Let $\delta \in \Delta$, let $I^{\delta} \subseteq \delta$, and let $c_{\delta} \in L(A)^{I^{\delta}}$ be a preference profile. Let for all parties $x, y \in A$ with $\pi_{c_{\delta}}(x) \neq 0$ and $\pi_{c_{\delta}}(y) \neq 0, \pi_{c_{\delta}}(x)=\pi_{c_{\delta}}(y)$. Then, for all parties with $\pi_{c_{\delta}}(x) \neq 0$ and $\pi_{c_{\delta}}(y) \neq 0, \pi_{c_{\delta}}(x)=\pi_{c_{\delta}}(y) \geq \pi_{c_{\delta}}(z)$ for all $z \in A$. Thus, for all parties $x, y \in A$ with $\pi_{c_{\delta}}(x) \neq 0$ and $\pi_{c_{\delta}}(y) \neq 0, \tau_{c_{\delta}}^{\Delta}(x)=\tau_{c_{\delta}}^{\Delta}(y)=1$ and, thus, $x, y \in t\left(F P T P_{\Delta}\left(c_{\delta}\right)\right)$. Also, for all $z \in A$ with $\pi_{c_{\delta}}(z)=0, \pi_{c_{\delta}}(z)<$ $\pi_{c_{\delta}}(x)=\pi_{c_{\delta}}(y)$ for all $x, y \in A$ with $\pi_{c_{\delta}}(x) \neq 0$ and $\pi_{c_{\delta}}(y) \neq 0$. Thus, for all parties $z \in A$ with $\pi_{c_{\delta}}(z)=0, \tau_{c_{\delta}}^{\Delta}(z)=0$ and, thus, $z \notin t\left(F P T P_{\Delta}\left(c_{\delta}\right)\right)$. Hence, $t\left(F P T P_{\Delta}\left(c_{\delta}\right)\right)=\left\{x \mid \pi_{c_{\delta}}(x) \neq 0\right\}$.

Finally, we define a property called district cancellation. A social preference rule has the district cancellation property if it declares a tie between party $x$ and party $y$ in case the number of constituencies in which $x$ is preferred most by the largest number of voters equals the number of constituencies in which $y$ is preferred most by the largest number of voters.

Definition 8 (District Cancellation) A social preference rule $F_{\Delta}$ has the district cancellation property if, for all $I \subseteq V$, for every $c \in L(A)^{I}$, and for all $x, y \in A$, if $\left|\left\{\delta \in \Delta \mid x \in t\left(F_{\Delta}\left(c_{\delta}\right)\right)\right\}\right|=\left|\left\{\delta \in \Delta \mid y \in t\left(F_{\Delta}\left(c_{\delta}\right)\right)\right\}\right|$, then $x \sim_{F_{\Delta}(c)} y$.

Proposition $4 \mathrm{FPTP}_{\Delta}$ satisfies district cancellation.

Proof First note that for any $x \in A$, any $\delta \in \Delta$ and for any profile $c, x \in t\left(F P T P_{\Delta}\left(c_{\delta}\right)\right)$ iff $x$ is preferred most in $\delta$ given $c$. Now suppose that $\left|\left\{\delta \in \Delta \mid x \in t\left(F P T P_{\Delta}\left(c_{\delta}\right)\right)\right\}\right|$ $=\left|\left\{\delta \in \Delta \mid y \in t\left(F P T P_{\Delta}\left(c_{\delta}\right)\right)\right\}\right|$. Then $\tau_{c}^{\Delta}(x)=\tau_{c}^{\Delta}(y)$ and hence, by definition, $x \sim_{F P T P}(c) y$.

In Sect.7, we shall see that the Plurality ranking rule $P l$ satisfies all the axioms mentioned so far except district cancellation, as shown by the following example.

Counterexample $3 A=\{x, y, z\}, \Delta=\{\delta\}, \delta=\{1,2,3\}, 1: x \ldots ; 2: x \ldots$; and 3 : $y \ldots$ Then $t\left(P l\left(c_{\delta}\right)\right)=\{x\}$. Hence, the number of districts with $y$ at the top of $P l\left(c_{\delta}\right)$ is 0 . The same holds for $z$, but not $y \sim_{P l\left(c_{\delta}\right)} z: y$ has more first votes than $z$.

\section{A Characterization of FPTP systems}

In this section, we prove that a social preference rule is $F P T P_{\Delta}$, if and only if, it satisfies subset consistency, district consistency, subset cancellation and district cancellation. In our proof, we use the following lemma.

Lemma 1 Let $F_{\Delta}: L(A)^{I} \rightarrow W(A)$. If $F_{\Delta}$ satisfies subset consistency and subset cancellation, then for every $\delta \in \Delta$ with $I^{\delta}=\delta \cap I \neq \emptyset, x \in t\left(F_{\Delta}\left(c_{\delta}\right)\right)$, if and only if, $\pi_{c_{\delta}}(x) \geq \pi_{c_{\delta}}(z)$ for all $z \in A$.

Proof First, we show that $t\left(F_{\Delta}\left(c_{\emptyset}\right)\right)=A$. Let $c_{\delta}$ be such that $\pi_{c_{\delta}}(x)=\pi_{c_{\delta}}(y)>0$ for all $x, y$. By subset cancellation, $t\left(F_{\Delta}\left(c_{\delta}\right)\right)=A$. Note that $c_{\delta}=c_{\delta}+c_{\emptyset}$ and $\delta \cap \emptyset=\emptyset$. By definition, $t\left(F_{\Delta}\left(c_{\emptyset}\right)\right) \neq \emptyset$. Hence, $t\left(F_{\Delta}\left(c_{\emptyset}\right)\right) \cap t\left(F_{\Delta}\left(c_{\delta}\right)\right) \neq \emptyset$, and by subset consistency, $t\left(F_{\Delta}\left(c_{\delta}+c_{\emptyset}\right)\right)=t\left(F_{\Delta}\left(c_{\delta}\right)\right) \cap t\left(F_{\Delta}\left(c_{\emptyset}\right)\right)$; that is $t\left(F_{\Delta}\left(c_{\delta}\right)\right)=$ $A=t\left(F_{\Delta}\left(c_{\emptyset}\right)\right)$. 
(1) if-part: We prove this part by induction on the number of voters $\left|I^{\delta}\right|=n$. For $n=1$, there is only one voter $i \in I^{\delta}$. If $\pi_{c_{\delta}}(x) \geq \pi_{c_{\delta}}(z)$ for all $z \in A$, then $x=t\left(c_{i}\right)$, and by subset cancellation, we have $x \in t\left(F_{\Delta}\left(c_{i}\right)\right)$.

Suppose that the assertion holds for all $I^{\delta}$ with $\left|I^{\delta}\right| \leq n$ and let $I^{\delta} \subseteq \delta \subseteq V$ be such that $\left|I^{\delta}\right|=n+1$. Let $x \in A$ with $\pi_{c_{\delta}}(x) \geq \pi_{c_{\delta}}(z)$ for all $z \in A$. Let $y_{1}, y_{2}, \ldots, y_{k} \in A$ be all parties with $\pi_{c_{\delta}}(x)=\pi_{c_{\delta}}\left(y_{j}\right), j=1, \ldots, k$. Let $I_{1}^{\delta}=\left\{i, i_{1}, i_{2}, \ldots, i_{k}\right\} \subseteq I^{\delta}$ such that $t\left(c_{i}\right)=x$ and $t\left(c_{i_{j}}\right)=y_{j}$. Let $I_{2}^{\delta}=I^{\delta} \backslash I_{1}^{\delta}$, so that $I^{\delta}=I_{1}^{\delta} \cup I_{2}^{\delta}$ and $I_{1}^{\delta} \cap I_{2}^{\delta}=\emptyset$. The restrictions of $c_{\delta}$ to $I_{1}^{\delta}$ and $I_{2}^{\delta}$ are $c_{\delta}^{1}$ and $c_{\delta}^{2}$.

Since $\left|I_{2}^{\delta}\right| \leq n$ and $\pi_{c_{\delta}^{2}}(x) \geq \pi_{c_{\delta}^{2}}(z)$ for all $z \in A$, by the induction hypothesis we have $x \in t\left(F_{\Delta}\left(c_{\delta}^{2}\right)\right)$. (In case $I_{2}^{\delta}=\emptyset, c_{\delta}^{2}=c_{\emptyset}$ and $t\left(F_{\Delta}\left(c_{\delta}^{2}\right)\right)=t\left(F_{\Delta}\left(c_{\emptyset}\right)\right)=$ A.) By subset cancellation, we have $x \in t\left(F_{\Delta}\left(c_{\delta}^{1}\right)\right)$. Hence, $t\left(F_{\Delta}\left(c_{\delta}^{1}\right)\right) \cap t\left(F_{\Delta}\left(c_{\delta}^{2}\right)\right) \neq \emptyset$ which gives, by subset consistency, $x \in t\left(F_{\Delta}\left(c_{\delta}\right)\right)=$ $t\left(F_{\Delta}\left(c_{\delta}^{1}+c_{\delta}^{2}\right)\right)=t\left(F_{\Delta}\left(c_{\delta}^{1}\right)\right) \cap t\left(F_{\Delta}\left(c_{\delta}^{2}\right)\right)$.

(2) only if part: Suppose that there exists a $z \in A$ with $z \in t\left(F_{\Delta}\left(c_{\delta}\right)\right)$ and $\pi_{c_{\delta}}(y)>$ $\pi_{c_{\delta}}(z)$ for some $y \in A$. Choose $x \in A$ such that $\pi_{c_{\delta}}(x) \geq \pi_{c_{\delta}}(z)$ for all $z \in A$. Let $I_{1}^{\delta}, I_{2}^{\delta}, c_{\delta}^{1}$ and $c_{\delta}^{2}$ be defined as in part (1). Then, by the result of part 1, we have $x \in t\left(F_{\Delta}\left(c_{\delta}^{2}\right)\right)$ and $x \in t\left(F_{\Delta}\left(c_{\delta}^{1}\right)\right)$. By subset cancellation, we have that $z \notin t\left(F_{\Delta}\left(c_{\delta}^{1}\right)\right)$. By subset consistency, it follows that $z \notin t\left(F_{\Delta}\left(c_{\delta}\right)\right)$. Contradiction.

Theorem 1 Let $F_{\Delta}: L(A)^{I} \rightarrow W(A)$, for $I \subseteq V$, be a social preference rule. Then $F_{\Delta}$ is FPT $P_{\Delta}$, if and only if, $F_{\Delta}$ is subset consistent and district consistent and has the subset cancellation property and the district cancellation property.

The 'only if' part is established by Propositions 1,2, 3 and 4.

Proof of the 'if' part: The proof of the if-part of the equivalence resembles in some respects the proof of Theorem 1 in Hout (2006). Let $F_{\Delta}: L(A)^{I} \rightarrow W(A)$, for $I \subseteq V$, be a social preference rule that satisfies these conditions. Let $I \subseteq V$ and let $c \in L(A)^{I}$. We should prove that for all $x, y \in A$,

$x \succ_{F_{\Delta}(c)} y$, iff, $x \succ_{F P T P_{\Delta}(c)} y$, i.e. iff $\tau_{c}^{\Delta}(x)>\tau_{c}^{\Delta}(y)$ and

$x \sim_{F_{\Delta}(c)} y$, iff, $x \sim_{F P T P_{\Delta}(c)} y$, i.e. iff $\tau_{c}^{\Delta}(x)=\tau_{c}^{\Delta}(y)$.

It is sufficient to show that

(1) If $\tau_{c}^{\Delta}(x)=\tau_{c}^{\Delta}(y)$, then $x \sim_{F_{\Delta}(c)} y$. Thus, if $\mid\left\{\delta \cap I \in \Delta^{I} \mid x\right.$ is preferred most in district $\delta$ given $c\}|=|\left\{\delta \cap I \in \Delta^{I} \mid y\right.$ is preferred most in district $\delta$ given $\left.c\right\} \mid$, then $x \sim F_{\Delta}(c) y$.

(2) If $\tau_{c}^{\Delta}(x)>\tau_{c}^{\Delta}(y)$, then $x \succ_{F_{\Delta}(c)} y$. Thus, if $\mid\left\{\delta \cap I \in \Delta^{I} \mid x\right.$ is preferred most in district $\delta$ given $c\}|>|\left\{\delta \cap I \in \Delta^{I} \mid y\right.$ is preferred most in district $\delta$ given c\} |, then $x \succ_{F_{\Delta}}(c) y$.

For suppose $x \sim_{F_{\Delta}(c)} y$ and not $\tau_{c}^{\Delta}(x) \neq \tau_{c}^{\Delta}(y)$, then either $\tau_{c}^{\Delta}(x)>\tau_{c}^{\Delta}(y)$ or $\tau_{c}^{\Delta}(x)<\tau_{c}^{\Delta}(y)$. If $\tau_{c}^{\Delta}(x)>\tau_{c}^{\Delta}(y)$, then by (2) $x>_{F_{\Delta}(c)} y$. If $\tau_{c}^{\Delta}(x)<\tau_{c}^{\Delta}(y)$, then by (2), $y \succ_{F_{\Delta}(c)} x$. This is a contradiction. Hence, if $x \sim_{F_{\Delta}(c)} y$, then $\tau_{c}^{\Delta}(x)=\tau_{c}^{\Delta}(y)$. Similarly, if $x \succ_{F_{\Delta}(c)} y$, then $\tau_{c}^{\Delta}(x)>\tau_{c}^{\Delta}(y)$.

Proof of (1): Suppose $\tau_{c}^{\Delta}(x)=\tau_{c}^{\Delta}(y)$. Then $\left|\left\{\delta \in \Delta \mid x \in t\left(F_{\Delta}\left(c_{\delta}\right)\right)\right\}\right|=\mid\{\delta \in \Delta \mid$ $\left.y \in t\left(F_{\Delta}\left(c_{\delta}\right)\right)\right\} \mid$. Hence, by district cancellation, $x \sim_{F_{\Delta}(c)} y$. 
Proof of (2): Suppose $\tau_{c}^{\Delta}(x)>\tau_{c}^{\Delta}(y)$. Then there are $I^{\prime}, I^{\prime \prime} \subseteq I$ such that:

$-\quad I=I^{\prime} \cup I^{\prime \prime}, I^{\prime} \cap I^{\prime \prime}=\emptyset$.

- for $c^{\prime}$ being the restriction of $c$ to $I^{\prime}, \tau_{c^{\prime}}^{\Delta}(x)=\tau_{c^{\prime}}^{\Delta}(y)$.

- $c^{\prime \prime}$, being the restriction of $c$ to $I^{\prime \prime}$, is such that for all $\delta \in \Delta$ with $\delta \cap I^{\prime \prime} \neq \emptyset$, $\pi_{c_{\delta}^{\prime \prime}}(x) \geq \pi_{c_{\delta}^{\prime \prime}}(z)$ for all $z \in A$ and $\pi_{c_{\delta}^{\prime \prime}}(x)>\pi_{c_{\delta}^{\prime \prime}}(y)$.

Notice that $I^{\prime \prime} \neq \varnothing$. By district cancellation, $x \sim_{F_{\Delta}\left(c^{\prime}\right)} y$ (i).

By Lemma 1, for every $\delta \in \Delta$ with $\delta \cap I^{\prime \prime} \neq \emptyset, x \in t\left(F_{\Delta}\left(c_{\delta}^{\prime \prime}\right)\right)$, if and only if, $\pi_{c_{\delta}^{\prime \prime}}(x) \geq \pi_{c_{\delta}^{\prime \prime}}(z)$ for all $z \in A$. This means that for every such $\delta, x \in t\left(F_{\Delta}\left(c_{\delta}^{\prime \prime}\right)\right)$ and $y \notin t\left(F_{\Delta}\left(c_{\delta}^{\prime \prime}\right)\right)$, since, for every such $\delta, \pi_{c_{\delta}^{\prime \prime}}(x) \geq \pi_{c_{\delta}^{\prime \prime}}(z)$ for all $z \in A$ and $\pi_{c_{\delta}^{\prime \prime}}(x)>\pi_{c_{\delta}^{\prime \prime}}(y)$. Thus, for each such $\delta, x \succ_{F_{\Delta}\left(c_{\delta}^{\prime \prime}\right)} y$. This means that, by district consistency, $x \succ_{F_{\Delta}\left(c^{\prime \prime}\right)} y$ (ii). Given (i) and (ii), by district consistency, $x \succ_{F_{\Delta}(c)} y$.

\section{Deeper axioms for social preference rules}

In this section, we introduce a number of properties which together imply the subset cancellation property (Lemma 2); for that reason, we speak of deeper axioms. Anonymity means that it does not matter who casts which vote; the names of the voters are irrelevant. In other words, all voters are treated equally.

Definition 9 (Anonymity) $F_{\Delta}$ is anonymous $:=$ for all $I \subseteq V$, for every permutation $\sigma$ of $I$, and for all preference profiles $c \in L(A)^{I}, F_{\Delta}(c \circ \sigma)=F_{\Delta}(c)$.

Clearly, $P l$ is anonymous, but $F P T P_{\Delta}$ is not, as shown by the following example.

Counterexample 4 Let $A=\{x, y, z\}, I=V=\{1, \ldots, 6\}, \Delta=\left\{\delta_{1}, \delta_{2}\right\}$ with $\delta_{1}=$ $\{1,2,3\}$ and $\delta_{2}=\{4,5,6\}, \sigma(1)=4$ and $\sigma(4)=1$. Let profile $c$ be as indicated in the table below.

$$
\begin{aligned}
& \frac{c \quad c \circ \sigma}{1 x \ldots y \ldots} \\
& \delta_{1} 2 x \ldots x \ldots \\
& 3 y \ldots y \ldots \\
& 4 y \ldots x \ldots \\
& \delta_{2} 5 z \ldots z \ldots \\
& 6 z \ldots z \ldots
\end{aligned}
$$

$\tau_{c}^{\Delta}(x)=1$ and $\tau_{c}^{\Delta}(y)=0$ and $\tau_{c}^{\Delta}(z)=1$. But $\tau_{c \circ \sigma}^{\Delta}(x)=0$ and $\tau_{c \circ \sigma}^{\Delta}(y)=1$ and $\tau_{c \circ \sigma}^{\Delta}(z)=1$. Hence, FPTP $P_{\Delta}(c \circ \sigma) \neq F P T P_{\Delta}(c)$.

Hence, FPTP is not anonymous, but it is clearly subset anonymous. A social preference rule is called subset anonymous if it treats all voters equally in case society consists of (a subset of) one single constituency. 
Definition 10 (Subset Anonymity). A social preference rule $F_{\Delta}$ is subset anonymous if, for all $\delta \in \Delta$, for all $I^{\delta} \subseteq \delta$, for every permutation $\sigma$ of $I^{\delta}$, and for all preference profiles $c_{\delta} \in L(A)^{I^{\delta}}, F_{\Delta}\left(c_{\delta} \circ \sigma\right)=F_{\Delta}\left(c_{\delta}\right)$.

Proposition $5 \mathrm{FPTP}_{\Delta}$ is subset anonymous.

Proof For all $\delta \in \Delta$, for all $I^{\delta} \subseteq \delta$, for every permutation $\sigma$ of $I^{\delta}$, for all preference profiles $c_{\delta} \in L(A)^{I^{\delta}}$, and for all $x \in A$, for all $i \in I^{\delta}, \pi_{(\operatorname{co\sigma })_{i}}(x)=\pi_{c_{\sigma(i)}}(x)$. Hence, for all $\delta \in \Delta$ and for all $x \in A, \pi_{c_{\delta} \circ \sigma}(x)=\pi_{c_{\delta}}(x)$ and, thus, $\tau_{c_{\delta} \circ \sigma}^{\Delta}=1$, iff, $\tau_{c_{\delta}}^{\Delta}(x)=1$. Hence, FPTP $P_{\Delta}\left(c_{\delta} \circ \sigma\right)=F P T P_{\Delta}\left(c_{\delta}\right)$.

We are able to show that subset cancellation is implied by subset anonymity, neutrality, topsonlyness, and Pareto optimality. Neutrality and topsonlyness are defined as usual. Neutrality requires that all parties are treated equally, where topsonlyness requires that whenever for two profiles the tops of the individual preference orderings correspond, the social outcome should be the same for both profiles.

Definition 11 (Neutrality) A social preference rule $F_{\Delta}$ is neutral if, for every permutation $\lambda$ of $A$, for all $I \subseteq V$, and for every preference profile $c \in L(A)^{I}, F_{\Delta}(\lambda c)=$ $\lambda F_{\Delta}(c)$.

\section{Proposition $6 \mathrm{FPTP}_{\Delta}$ is neutral.}

Proof For every permutation $\lambda$ of $A$, for all $I \in V$, for every preference profile $c \in$ $L(A)^{I}$, and for all $x \in A, \tau_{\lambda c}^{\Delta}(\lambda(x))=\tau_{c}^{\Delta}(x)$. Thus, $F P T P_{\Delta}(\lambda c)=\lambda F P T P_{\Delta}(c)$.

Definition 12 (Topsonlyness) A social preference rule $F_{\Delta}$ is topsonly if, whenever $c, c^{\prime} \in L(A)^{I}$ are such that for all $i \in I$ and for all $x \in A, t\left(c_{i}\right)=x$ iff $t\left(c_{i}^{\prime}\right)=x$, $F_{\Delta}(c)=F_{\Delta}\left(c^{\prime}\right)$.

Proposition $7 \mathrm{FPTP}_{\Delta}$ is topsonly.

Proof Suppose that $c, c^{\prime} \in L(A)^{I}$ are such that for all $i \in I$ and for all $x \in A$, $t\left(c_{i}\right)=x$ iff $t\left(c_{i}^{\prime}\right)=x$. Then for all $\delta \in \Delta$ and for all $x \in A, \pi_{c_{\delta}}(x)=\pi_{c_{\delta}^{\prime}}(x)$ and, thus, for all $x \in A, \tau_{c}^{\Delta}(x)=\tau_{c^{\prime}}^{\Delta}(x)$. Hence, $\operatorname{FPTP}_{\Delta}(c)=\operatorname{FPTP}_{\Delta}\left(c^{\prime}\right)$.

Pareto optimality requires that, whenever all individuals prefer $x$ to $y$, then $y$ is not ranked first socially.

Definition 13 (Pareto optimality) A social preference rule $F_{\Delta}$ is Pareto optimal if, for all parties $x, y \in A$, for all $I \subseteq V$, and for all preference profiles $c \in L(A)^{I}$ : if $x \succ_{c_{i}} y$ for all $i \in I$, then $y \notin t\left(F_{\Delta}(c)\right)$.

Proposition $8 \mathrm{FPTP}_{\Delta}$ is Pareto optimal.

Proof Let $x, y \in A$, let $I \subseteq V$, and let $c \in L(A)^{I}$ be such that $x \succ_{c_{i}} y$ for all $i \in I$. Then for all $i \in I, t\left(c_{i}\right) \neq y$. Hence, for each $\delta \in \Delta$ there is some $z^{\delta} \in A, z^{\delta} \neq y$ such that $\pi_{c_{\delta}}\left(z^{\delta}\right)>\pi_{c_{\delta}}(y)$, and, thus $\tau_{c_{\delta}}^{\Delta}(y)=0$. Hence, there is some party $v \in A$, $v \neq y$, such that $\tau_{c}^{\Delta}(v)>\tau_{c}^{\Delta}(y)$ and, thus, $y \notin t\left(F P T P_{\Delta}(c)\right)$. 
Lemma 2 If $F_{\Delta}$ is subset anonymous, neutral, topsonly, and Pareto optimal, then $F_{\Delta}$ has the subset cancellation property.

Proof Let $F_{\Delta}$ be subset anonymous, neutral, topsonly, and Pareto optimal. Let $\delta \in \Delta$ and let $c_{\delta} \in L(A)^{I^{\delta}}$ be such that for all parties $x, y \in A$ with $\pi_{c_{\delta}}(x) \neq 0$ and $\pi_{c_{\delta}}(y) \neq$ $0, \pi_{c_{\delta}}(x)=\pi_{c_{\delta}}(y)$. We should prove that $t\left(F_{\Delta}\left(c_{\delta}\right)\right)=\left\{x \in A \mid \pi_{c_{\delta}}(x) \neq 0\right\}$.

For a set of parties $\{x, y\} \subseteq A$ with $\pi_{c_{\delta}}(x) \neq 0$ and $\pi_{c_{\delta}}(y) \neq 0$, let $1,2, \ldots, k$ be the voters that voted for $x$ and let $k+1, \ldots 2 k$ be the voters that voted for $y$. Let $\sigma$ be the permutation of $I^{\delta}$ in which for all $i \in\{1, \ldots, 2 k\}, \sigma(i)=2 k-(i-1)$, and for all other voters $\sigma(i)=i$. Since $F_{\Delta}$ is subset anonymous, we have

$$
F_{\Delta}\left(c_{\delta} \circ \sigma\right)=F_{\Delta}\left(c_{\delta}\right)
$$

Let $\lambda$ be the permutation of $A$ with $\lambda(x)=y, \lambda(y)=x$ and $\lambda(z)=z$ for all $z \in A$, $z \neq x, z \neq y$. Since $F_{\Delta}$ is neutral, it follows that

$$
F_{\Delta}\left(\lambda c_{\delta}\right)=\lambda F_{\Delta}\left(c_{\delta}\right)
$$

Since $c_{\delta} \circ \sigma$ and $\lambda c_{\delta}$ are such that, for all $i \in I^{\delta}$ and for all $x \in A, t(c \circ \sigma)_{i}=x$ iff $t\left(\lambda c_{i}\right)=x$, by topsonlyness we have

$$
F_{\Delta}\left(c_{\delta} \circ \sigma\right)=F_{\Delta}\left(\lambda c_{\delta}\right)
$$

By (1), (2) and (3) it follows that

$$
F_{\Delta}\left(c_{\delta}\right)=\lambda F_{\Delta}\left(c_{\delta}\right)
$$

Hence, for each set of parties $\{x, y\} \subseteq A$ with $\pi_{c_{\delta}}(x) \neq 0$ and $\pi_{c_{\delta}}(y) \neq 0$, we have that

$$
x \in t\left(F_{\Delta}\left(c_{\delta}\right)\right) \text { iff } y \in t\left(F_{\Delta}\left(c_{\delta}\right)\right)
$$

Now there are two cases:

1. There are no parties $z \in A$ with $\pi_{c_{\delta}}(z)=0$. This means that $t\left(F\left(c_{\delta}\right)\right)=\{x \mid$ $\left.\pi_{x}\left(c_{\delta}\right) \neq 0\right\}$.

2. There is at least one party $z \in A$ with $\pi_{c_{\delta}}(z)=0$. In this case, it is possible to construct a profile $c_{\delta}^{\prime} \in L(A)^{I^{\delta}}$ such that $t\left(c_{\delta i}^{\prime}\right)=t\left(c_{\delta i}\right)$ for all $i \in I^{\delta}$ and such that there exists a party $x$ such that for all parties $z \in A$ with $\pi_{c_{\delta}}(z)=0, x \succ_{c_{\delta i}^{\prime}} z$ for all $i \in I^{\delta}$. By topsonlyness, we have

$$
F_{\Delta}\left(c_{\delta}^{\prime}\right)=F_{\Delta}\left(c_{\delta}\right)
$$

By Pareto optimality, we have for all parties $z \in A$ with $\pi_{c_{\delta}}(z)=0$

$$
z \notin t\left(F_{\Delta}\left(c_{\delta}^{\prime}\right)\right)
$$

By (5), (6) and (7), it follows that $t\left(F_{\Delta}\left(c_{\delta}\right)\right)=\left\{x \mid \pi_{c_{\delta}}(x) \neq 0\right\}$. 
Theorem 2 Let $F_{\Delta}: L(A)^{I} \rightarrow W(A)$, for $I \subseteq V$, be a social preference rule. Then $F_{\Delta}$ is FPT $P_{\Delta}$, if and only if, $F_{\Delta}$ is subset consistent, district consistent, subset anonymous, neutral, topsonly, Pareto optimal, and has the district cancellation property.

Proof The 'only if' part is shown by Propositions 1-8. In Lemma 2 it was shown that subset anonymity, neutrality, topsonlyness, and Pareto optimality imply the subset cancellation property. By Theorem1, subset cancellation, district cancellation, subset consistency, and district consistency imply an FPTP-system.

\section{Independence of the properties}

The properties mentioned in Theorem 1 are independent.

Proposition 9 Subset cancellation, district cancellation, subset consistency, and district consistency are independent.

Proof A function that does not satisfy subset cancellation, but does satisfy the other properties is the function $F 1_{\Delta}: L(A)^{I} \rightarrow W(A)$, for $I \subseteq V$, defined by $x \succeq_{F 1_{\Delta}(c)} y$, iff, $\beta_{c}^{\Delta}(x) \geq \beta_{c}^{\Delta}(y)$, where $\beta_{c}^{\Delta}(x):=\mid\left\{\delta \cap I \in \Delta^{I} \mid\right.$ Borda score $\left(c_{\delta}, x\right) \geq$ Borda score $\left(c_{\delta}, y\right)$ for all $\left.y \in A\right\} \mid$.

A function that satisfies all properties but district cancellation is the function $F 2_{\Delta}$ : $L(A)^{I} \rightarrow W(A)$, for $I \subseteq V$, defined by $x \succeq_{F 2_{\Delta}(c)} y$, iff, district Borda score $(c, x)$ given $\Delta \geq$ district Borda score $(c, y)$ given $\Delta$. Here, district Borda score $(c, x)$ given $\Delta:=$ a score of $m-a, m$ being the number of parties, each time $x$ is preferred most by the $a$ th most voters in a particular district $\delta$, summed over all districts $\delta \in \Delta$ with $\delta \cap I \neq \emptyset$.

A function that satisfies all properties but subset consistency is the function $F 3_{\Delta}$ : $L(A)^{I} \rightarrow W(A)$, for $I \subseteq V$, defined by $x \succeq_{F_{3_{\Delta}}(c)} y$, iff, $\gamma_{c}^{\Delta}(x) \geq \gamma_{c}^{\Delta}(y)$, where $\gamma_{c}^{\Delta}(x):=\mid\left\{\delta \cap I \in \Delta^{I} \mid \pi_{c_{\delta}}(x) \neq 0\right.$ and $\pi_{c_{\delta}}(z) \geq \pi_{c_{\delta}}(x)$ for all $z \in A$ with $\left.\pi_{c_{\delta}}(z) \neq 0\right\} \mid$.

In order to describe a function that fails to satisfy district consistency, we consider the set of parties that are preferred most in the largest number of constituencies. A function that satisfies all properties but district consistency is the function that ranks these parties first and ranks all other parties second. In order to give a formal description of this rule, consider $X_{1}:=\left\{x \in A \mid \forall y \neq x\left[\tau_{c_{\delta}}^{\Delta}(x) \geq \tau_{c_{\delta}}^{\Delta}(y)\right]\right\}$.

The function that does not satisfy district consistency is the function $F 4_{\Delta}: L(A)^{I}$ $\rightarrow W(A)$, for $I \subseteq V$, defined by: (i) for all $x, y \in X_{1}, x \sim_{F 4_{\Delta}(c)} y$, (ii) for all $z, u \in A \backslash X_{1}, z \sim_{F 4_{\Delta}(c)} u$, (iii) for all $x \in X_{1}$ and $z \in A \backslash X_{1}, x \succ_{F 4_{\Delta}(c)} z$.

Also the properties mentioned in Theorem 2 are independent.

Proposition 10 Subset consistency, district consistency, subset anonymity, neutrality, topsonlyness, Pareto optimality and the district cancellation property are independent. 
Proof The function $F 3_{\Delta}$ satisfies all these properties but subset consistency.

A function that satisfies all properties but district consistency is the function $F 4_{\Delta}$.

A function that does not satisfy subset anonymity but does satisfy the other axioms is the function that counts in each district the vote of one particular voter twice. Formally, for each $\delta \in \Delta$, let $j_{\delta}$ be the largest element in $\delta$ and define for all $x \in A$, for $i \in \delta$, (i) $\pi_{c_{i}}^{\prime}(x)=2$ if $i=j_{\delta}$ and $t\left(c_{i}\right)=x$, (ii) $\pi_{c_{i}}(x)=1$ if $i \neq j_{\delta}$ and $t\left(c_{i}\right)=x$, (iii) $\pi_{c_{i}}=0$ in all other cases. The function that is not subset anonymous is the function $F 5_{\Delta}: L(A)^{I} \rightarrow W(A)$, for $I \subseteq V$, defined by $x \succ_{F 5_{\Delta}(c)} y$, iff, $\epsilon_{c}^{\Delta}(x)>\epsilon_{c}^{\Delta}(y)$ and $x \sim_{F 5_{\Delta}(c)} y$, iff, $\epsilon_{c}^{\Delta}(x)=\epsilon_{c}^{\Delta}(y)$, where $\epsilon_{c}^{\Delta}(x):=\mid\left\{\delta \cap I \in \Delta^{I} \mid \pi_{c \delta}^{\prime}(x) \geq \pi_{c_{\delta}}^{\prime}(y)\right.$ for all $y \in A\} \mid$.

A function that is not neutral is the function that differs from $F P T P_{\Delta}$ in the fact that for a certain party $z$, party $z$ is ranked before the other parties if party $z$ is preferred most in an absolute majority of the constituencies, or else this party $z$ is ranked last. Formally, this is a function $F 6_{\Delta}: L(A)^{I} \rightarrow W(A)$, for $I \subseteq V$, defined by, for $z \in A$, if $\tau_{c}^{\Delta}(z)>1 / 2|\Delta|$, then $z \succ_{F 6_{\Delta}} y$ for all $y \in A, y \neq z$, if $\tau_{c}^{\Delta}(z) \leq 1 / 2|\Delta|$, then $z \prec_{F 6_{\Delta}} y$ for all $y \in A, y \neq z$,

and for $x, y \in A, x \neq z, y \neq z, x \succ_{F 6_{\Delta}} y$, iff, $\tau_{c}^{\Delta}(x)>\tau_{c}^{\Delta}(y)$ and $x \sim_{F 6_{\Delta}} y$, iff, $\tau_{c}^{\Delta}(x)=\tau_{c}^{\Delta}(y)$.

A function that satisfies all the properties but topsonlyness is the function $F 1_{\Delta}$.

A function that is not Pareto optimal, while satisfying all other properties, is the function $F 7_{\Delta}: L(A)^{I} \rightarrow W(A)$, for $I \subseteq V$, defined by $x>_{F 7_{\Delta}} y$, iff, $\tau_{c}^{\Delta}(x)<\tau_{c}^{\Delta}(y)$ and $x \sim_{F 7_{\Delta}} y$, iff, $\tau_{c}^{\Delta}(x)=\tau_{c}^{\Delta}(y)$.

Finally, a function that satisfies all properties but district cancellation is the function $F 2_{\Delta}$.

\section{FPTP and list PR compared}

Now that we have found the characteristic properties for FPTP systems modelled as social preference rules, we may compare these with the characteristic properties that we found for list PR systems, also modelled as social preference rules, that we found in Hout (2006). In order to do so, we will specify how the sets of characteristic properties that were found for both systems, modelled as social preference rules, relate to each other. We will indicate which properties distinguish FPTP systems from list PR systems and, on the other hand, what properties distinguish list PR systems from FPTP systems.

In Hout (2006), we found that list PR systems, modelled as the plurality ranking rule $P l$, are characterized by consistency (Con), faithfulness (F), anonymity (A), neutrality $(\mathrm{N})$ and topsonlyness $(\mathrm{T})$. This is indicated in Table 1, where the characteristic properties have been printed in boldface.

Faithfulness requires of a decision rule that it chooses according to the individual preference ordering in a one-person situation. A social preference rule will be called faithful if, in case society consists of a single individual whose most preferred party is party $x$, it orders this party $x$ first.

Definition 14 (Faithfulness) Let $\{i\} \subseteq V$ be a set of voters consisting of a single individual. A social preference rule $F_{\Delta}$ is faithful if, for all $i \in N$, for all $c_{i} \in L(P)^{\{i\}}$, and for all $x \in A$, if $t\left(c_{i}\right)=x$, then $t\left(F_{\Delta}\left(c_{i}\right)\right)=x$. 
Table 1 Properties of list PR systems and FPTP systems

\begin{tabular}{lllllllllll}
\hline & Con & SCon & DCon & F & A & SA & N & T & PO & DCan \\
\hline List PR & $\mathbf{1}$ & 1 & 1 & $\mathbf{1}$ & $\mathbf{1}$ & 1 & $\mathbf{1}$ & $\mathbf{1}$ & 1 & \\
FPTP & & $\mathbf{1}$ & $\mathbf{1}$ & 1 & & $\mathbf{1}$ & $\mathbf{1}$ & $\mathbf{1}$ & $\mathbf{1}$ & $\mathbf{1}$ \\
\hline
\end{tabular}

In this article, we found that characteristic properties for FPTP systems are subset consistency (SCon), district consistency (DCon), subset anonymity (SA), neutrality (N), topsonlyness (T), Pareto optimality (PO), and district cancellation (DCan); see again Table 1 .

In this section, we will show that

(1) subset consistency (SCon) and district consistency (DCon) are implied by consistency (Con),

(2) Pareto optimality (PO) is implied by consistency (Con), faithfulness (F), anonymity (A), neutrality (N) and topsonlyness (T), all taken together, and

(3) subset anonymity (SA) is implied by anonymity (A).

Hence, the Plurality ranking rule $P l$ satisfies all the characteristic properties of $F P T P_{\Delta}$ except district cancellation (DCan), as shown in Counterexample 3.

On the other hand, we will show that faithfulness $(\mathrm{F})$ is implied by Pareto optimality (PO). Hence, FPTP systems satisfies all characteristic properties of the Plurality ranking rule except consistency (Con) and anonymity (A), as shown in counterexample 1 and 4 respectively.

As we can read from Table 1, this means that anonymity (A) and consistency (Con) are the characteristic properties that distinguish list PR systems from FPTP systems. On the other hand, this means that district cancellation (DCan) is the characteristic property that distinguishes FPTP systems from list PR systems.

It is easy to verify that consistency implies district consistency. These are in fact very similar conditions, with the sole difference that district consistency implies additional requirements on the different sets of voters.

Proposition 11 If $F_{\Delta}$ is consistent (Con), then $F_{\Delta}$ is district consistent (DCon).

Proof By consistency, whenever $c \in L(A)^{I}, c^{\prime} \in L(A)^{J}$ are preference profiles for disjoint sets of voters $I \subseteq V$ and $J \subseteq V$ and $c+c^{\prime}$ is the profile on $I \cup J$ that corresponds with $c$ on $I$ and with $c^{\prime}$ on $J$, for all $x, y \in A$, if $x \succ_{F_{\Delta}(c)} y$ and $x \succeq_{F_{\Delta}\left(c^{\prime}\right)} y$, then $x \succ_{F_{\Delta}\left(c+c^{\prime}\right)} y$.

This means that this is certainly the case whenever $I \subseteq V$ and $J \subseteq V$ are such that for all $\delta \in \Delta, \delta \cap I \neq \emptyset$ implies $\delta \cap J=\emptyset$, and $c+c^{\prime}$ is the profile on $I \cup J$, that corresponds with $c$ on $I$ and with $c^{\prime}$ on $J$.

We are also able to show that, if we assume that disjoint subsets of constituencies do not share more than one element in the top of their social orderings, subset consistency (SCon) is implied by consistency (Con). 
Proposition 12 Let $I^{\delta} \subseteq \delta \subseteq V$ and $J^{\delta} \subseteq \delta \subseteq V$ be disjoint subsets of one single constituency $\delta$. Let for all $c_{\delta} \in L(A)^{I^{\delta}}$ and $c_{\delta}^{\prime} \in L(A)^{J^{\delta}}$ and for all $x, y \in A$, $x \in t\left(F_{\Delta}\left(c_{\delta}\right)\right)$ and $y \in t\left(F_{\Delta}\left(c_{\delta}\right)\right)$ implies that either $x \notin t\left(F_{\Delta}\left(c_{\delta}^{\prime}\right)\right)$ or $y \notin t\left(F_{\Delta}\left(c_{\delta}^{\prime}\right)\right)$. If $F_{\Delta}$ is consistent, then $F_{\Delta}$ is subset consistent.

Proof Let $I^{\delta} \subseteq \delta \subseteq V$ and $J^{\delta} \subseteq \delta \subseteq V$ be disjoint subsets of one single constituency $\delta$. Let $c_{\delta} \in L(A)^{I^{\delta}}$ and $c_{\delta}^{\prime} \in L(A)^{J^{\delta}}$ be preference profiles for $I^{\delta}$ and $J^{\delta}$, and $c_{\delta}+c_{\delta}^{\prime}$ be the profile on $I^{\delta} \cup J^{\delta}$ that corresponds with $c_{\delta}$ on $I^{\delta}$ and with $c_{\delta}^{\prime}$ on $J^{\delta}$. Suppose for all $c_{\delta} \in L(A)^{I^{\delta}}$ and $c_{\delta}^{\prime} \in L(A)^{J^{\delta}}$ and for all $x, y \in A, x \in t\left(F_{\Delta}\left(c_{\delta}\right)\right)$ and $y \in t\left(F_{\Delta}\left(c_{\delta}\right)\right)$ implies that either $x \notin t\left(F_{\Delta}\left(c_{\delta}^{\prime}\right)\right)$ or $y \notin t\left(F_{\Delta}\left(c_{\delta}^{\prime}\right)\right)(*)$. Suppose $t\left(F_{\Delta}\left(c_{\delta}\right)\right) \cap t\left(F_{\Delta}\left(c_{\delta}^{\prime}\right)\right) \neq \emptyset$ and suppose $F_{\Delta}$ is consistent. We should prove that $t\left(F_{\Delta}\left(c_{\delta}+c_{\delta}^{\prime}\right)\right)=t\left(F_{\Delta}\left(c_{\delta}\right)\right) \cap t\left(F_{\Delta}\left(c_{\delta}^{\prime}\right)\right)$.

We first show that $t\left(F_{\Delta}\left(c_{\delta}\right)\right) \cap t\left(F_{\Delta}\left(c_{\delta}^{\prime}\right)\right) \subseteq t\left(F_{\Delta}\left(c_{\delta}+c_{\delta}^{\prime}\right)\right)$. Let $x \in t\left(F_{\Delta}\left(c_{\delta}\right)\right) \cap$ $t\left(F_{\Delta}\left(c_{\delta}^{\prime}\right)\right)$. Then by (*), for all $z \in A$, either (i) $x \succ_{F_{\Delta}\left(c_{\delta}\right)} z$ and $x \succ_{F_{\Delta}\left(c_{\delta}^{\prime}\right)} z$, or (ii) $x \sim_{F_{\Delta}\left(c_{\delta}\right)} z$ and $x \succ_{F_{\Delta}\left(c_{\delta}^{\prime}\right)} z$, or (iii) $x \succ_{F_{\Delta}\left(c_{\delta}\right)} z$ and $x \sim_{F_{\Delta}\left(c_{\delta}^{\prime}\right)} z$.

In either case, by consistency, $x \succ_{F_{\Delta}\left(c_{\delta}+c_{\delta}^{\prime}\right)} z$. Hence, if $x \in t\left(F_{\Delta}\left(c_{\delta}\right)\right) \cap t\left(F_{\Delta}\left(c_{\delta}^{\prime}\right)\right)$, then $x \in t\left(F_{\Delta}\left(c_{\delta}+c_{\delta}^{\prime}\right)\right)$.

Conversely, suppose $z \in t\left(F_{\Delta}\left(c_{\delta}+c_{\delta}^{\prime}\right)\right)$. Let $x \in t\left(F_{\Delta}\left(c_{\delta}\right)\right) \cap t\left(F_{\Delta}\left(c_{\delta}^{\prime}\right)\right)$. If $z \notin t\left(F_{\Delta}\left(c_{\delta}\right)\right)$ or $z \notin t\left(F_{\Delta}\left(c_{\delta}^{\prime}\right)\right)$, then either (i) $x \succ_{F_{\Delta}\left(c_{\delta}\right)} z$ and $x \sim_{F_{\Delta}\left(c_{\delta}^{\prime}\right)} z$, or (ii) $x \sim_{F_{\Delta}\left(c_{\delta}\right)} z$ and $x \succ_{F_{\Delta}\left(c_{\delta}^{\prime}\right)} z$, or (iii) $x \succ_{F_{\Delta}\left(c_{\delta}\right)} z$ and $x \succ_{F_{\Delta}\left(c_{\delta}^{\prime}\right)} z$.

In either case, by consistency, $x \succ_{F\left(c_{\delta}+c_{\delta}^{\prime}\right)} z$. This contradicts $z \in t\left(F_{\Delta}\left(c_{\delta}+c_{\delta}^{\prime}\right)\right)$. Hence, $z \in t\left(F_{\Delta}\left(c_{\delta}\right)\right)$ and $z \in t\left(F_{\Delta}\left(c_{\delta}^{\prime}\right)\right)$.

Next we show that Pareto optimality is implied by consistency, faithfulness, anonymity, neutrality and topsonlyness. This follows from the fact that these conditions imply the plurality ranking rule and the plurality ranking rule in its turn satisfies Pareto optimality.

Proposition 13 If $F_{\Delta}$ is consistent, faithful, anonymous, neutral and topsonly, then $F_{\Delta}$ is Pareto optimal.

Proof In Hout (2006), it is shown that if $F_{\Delta}$ is consistent, faithful, anonymous, neutral and topsonly, then $F_{\Delta}$ is the plurality ranking rule $D . D$ satisfies Pareto optimality: Let $x, y \in A$, let $I \subseteq V$, and let $c \in L(A)^{I}$ be such that $x \succ_{c_{i}} y$ for all $i \in I$. Then $\pi_{c}(y)=0$ and $\pi_{c}(z)>0$ for at least one $z \in A, z \neq y$. Thus, $y \notin t(D(c))$.

It is easy to see that subset anonymity is implied by anonymity.

Proposition 14 If $F_{\Delta}$ is anonymous $(A)$, then $F_{\Delta}$ is subset anonymous $(S A)$.

Proof If for each set of voters $I$, and for every permutation $\sigma$ if $I, F_{\Delta}(c \circ \sigma)=F_{\Delta}(c)$, then certainly this is the case whenever $I=I^{\delta} \subseteq \delta$, with $\delta \in \Delta$.

Finally, it is also easy to verify that Faithfulness is implied by Pareto optimality.

Proposition 15 If $F_{\Delta}$ is Pareto optimal $(P O)$, then $F_{\Delta}$ is faithful $(F)$.

Proof If $I=\{i\}$ is a set of voters consisting of one single individual and $t\left(c_{i}\right)=x$, then for all $i \in I, x \succ_{c_{i}} y$ for all $y \in A, y \neq x$. Thus, by Pareto optimality, $y \notin t\left(F_{\Delta}\left(c_{i}\right)\right)$ for all $y \in A, y \neq x$. Hence, $t\left(F_{\Delta}\left(c_{i}\right)\right)=\{x\}$. 


\section{Conclusion}

In this article, we found characterizations for FPTP systems modelled as social preference rules. We were able to show that a social preference rule is an FPTP system, if and only if, it satisfies four properties: subset consistency and district consistency, the subset cancellation property and the district cancellation property. We also proved that subset cancellation is implied by four deeper properties, named as subset anonymity, neutrality, topsonlyness and Pareto optimality. This gives us a second characterization of an FPTP system.

Next, we compared the characteristic properties we found for FPTP systems to the characteristic properties of list PR systems, modelled as the plurality ranking rule, that we found in Hout (2006). We showed that list PR and FPTP systems have the following properties in common (see Table 1): subset consistency, district consistency, faithfulness, subset anonymity, neutrality, topsonlyness and Pareto optimality. Furthermore, we showed that consistency and anonymity are the characteristic properties of list PR systems that distinguish them from FPTP systems. The other way round, we showed that the characteristic property of FPTP systems that distinguishes them from list PR systems is district cancellation.

As a consequence, provided that we want a rule to satisfy all the properties that they share, a defence of list PR systems against FPTP systems should be based on an argument why consistency and anonymity are more important than district cancellation. Similarly, a defence of FPTP systems against list PR systems should be based on an argument why district cancellation is more important than consistency and anonymity, given that we want a rule to satisfy all the shared properties.

Acknowledgements The authors thank Rob Bosch, and two anonymous referees for their helpful and detailed comments.

Open Access This article is distributed under the terms of the Creative Commons Attribution Noncommercial License which permits any noncommercial use, distribution, and reproduction in any medium, provided the original author(s) and source are credited.

\section{References}

Farrell, D. M. (1997). Comparing electoral systems. Hertforshire: Prentice Hall/Harvester Wheatsheaf. van der Hout, E., de Swart, H., \& ter Veer, A. (2006). Characteristic properties of list PR systems. Social Choice and Welfare, 27, 459-475.

Lijphart, A. (1994). Electoral systems and party systems. A Study of 27 Democracies 1945-1990. New York: Oxford University Press.

Nitzan, S. \& Rubinstein, A. (1981). A further characterization of Borda ranking method. Public Choice, $36,153-158$.

Pitkin, H. F. (1967). The concept of representation. Berkely and Los Angeles: University of California Press.

Young, H. P. (1974). An axiomatization of Borda's rule. Journal of Economic Theory 9, 43-52. 